\title{
Role of laparoscopy in the Etiologic Diagnosis of Ascites of Unknown Origin
}

\author{
Sharma $A^{1}$, Thapa $P^{2}$, Gupta $\mathrm{SN}^{3}$
}

\begin{abstract}
Introduction: Ascites is a consequence of many different etiologies, such as liver cirrhosis, neoplasm, tuberculous peritonitis, pyogenic peritonitis, congestive heart failure, renal and pancreatic diseases but, in some situations, ascites is of unknown cause in spite of comprehensive study. The aim of this study was to identify the role of laparoscopy in the etiological diagnosis of ascites of unknown origin. Methods: This was a prospective study of the patients who underwent diagnostic laparoscopy to determine the causes of ascites of unknown origin in the Department of Surgery, Nepalgunj Medical College Teaching Hospital from April 2012 to May 2014. All the patients underwent laparoscopy for the evaluation of ascites after appropriate clinical and laboratory examinations, which failed to reveal the cause. Results: Peritoneal tuberculosis and carcinomatosis peritonei were the two most common causes found in $37.14 \%$ and $57.14 \%$ of cases respectively. The average age of the patients was 52 years. Distension of abdomen, abdominal pain and weight loss were the most frequently observed symptoms in 33 patients (100\%), 26 patients (74.28\%) and 18 patients (51.42\%) respectively. The CT scan findings, were a omental thickening in 28 cases (80\%), peritoneal nodules in $7(20 \%)$ patients and the intraabdominal lymph nodes in 13 patients (39.39\%). Ovarian mass was found in 4 patients (11.42\%). The histological diagnosis was a peritoneal carcinomatosis in 13 (37.14\%) patients and peritoneal tuberculosis in 20 (57.1\%) patients and in two patients nonspecific inflammation. The sensitivity and specificity of laparoscopic diagnosis in the diagnosis of peritoneal tuberculosis were $78.67 \%$ and $98.6 \%$ respectively and in the diagnosis of peritoneal carcinomatosis were $94.78 \%$ and $72.2 \%$ respectively. The positive predictive value was $97.3 \%$ and the negative predictive value was $73.7 \%$ for peritoneal tuberculosis and for peritoneal carcinomatosis the positive predictive value was $83.7 \%$ and negative predictive value was $94.87 \%$. Conclusion: The etiologic diagnosis of ascites of unknown origin is difficult despite the availability of several tests. Laparoscopy with peritoneal biopsy has still got a role in diagnosing these types of ascites where the other laboratory and imaging studies fail to reveal the cause.
\end{abstract}

Key words: Ascites, laparoscopy, peritoneal tuberculosis, peritoneal carcinomatosis

\section{INTRODUCTION}

Ascites is a consequence of many different underlying diseases. The standard procedure to assess ascites includes laboratory examinations (cell count, albumin level, total protein level, Gram stain, culture and cytology) and image investigations (ultrasound and computed tomography (CT) scan). The etiology of majority of ascites can be identified by these investigations. Ascites of unknown origin is defined as the etiology of ascites that cannot be determined after conventional laboratory examinations and imaging investigations. This poses a major diagnostic challenge for clinicians. The etiologies of these ascites, say of unknown origin, are dominated by tuberculosis and peritoneal carcinomatosis requiring early diagnosis and care ${ }^{1,2}$.

1. Dr. Anup Sharma

2. Dr. P. Thapa

3. Prof. S. N. Gupta

Address for correspondence:

Dr. Anup Sharma

Department of Surgery

Nepalgunj Medical College Teaching Hospital

Kohalpur, Banke, Nepal

Email: anupsharma88@yahoo.com
Laparoscopy as a minimally invasive technique has developed rapidly in recent years. Laparoscopy has long played an important role in the evaluation of ascites when its cause cannot be clarified clinically ${ }^{3}$. Although the utility of diagnostic laparoscopy has recently been questioned, possibly because of the advent of various non-invasive scanning techniques, ${ }^{4,5}$ it is a reliable technique for the investigation of patients presenting with ascites of unknown origin.

The aim of this work was to describe the value of diagnostic laparoscopy and histology in etiological diagnosis of ascites of unknown origin.

\section{MATERIAL AND METHODS}

It is a prospective study including all patients who underwent diagnostic laparoscopy to determine the causes of ascites in the Department of Surgery, Nepalgunj Medical College Teaching Hospital during the period from April 2012 to May 2014. All the patients underwent laparoscopy for the evaluation of ascites after appropriate clinical and laboratory examinations, including ascitic fluid for cytology as well as acid fast bacilli, serum albumin ascetic gradient,chest $x$-ray, ultrasonography or CT abdomen, had failed to reveal the cause. Procedures took place under general anesthesia. Patients with uncorrectable coagulopathy and severe cardiopulmonary diseases were excluded. 
Pneumoperitoneum was created by open technique through supraumbilical port. Laparoscopic examination was performed with either a $10 \mathrm{~mm} 0^{\circ}$ forward-viewing or a $10 \mathrm{~mm} 30^{\circ}$ oblique-viewing telescope. Biopsy of the suspicious lesion was performed through an additional $5 \mathrm{~mm}$ port. The visual diagnosis of tuberculous peritonitis was based on the presence of multiple yellowish white miliary tubercles of uniform size (usually $<5 \mathrm{~mm}$ ) on the visceral and parietal peritoneum.

Carcinomatosis peritonei was diagnosed by the presence of large nodules ( 1 to $5 \mathrm{~cm}$ in diameter) on the parietal peritoneum, omentum, falciform ligament or liver surface. Liver cirrhosis was diagnosed by the presence of irregular nodules on the liver surface, and collaterals on the falciform ligament and the greater omentum. The final diagnosis was made on the histopathological diagnosis. All data were analyzed using Statistical Package for Social Science (SPSS). Chisquare and t-tests were used to analyze data, as appropriate, and statistical significance was established at $p<0.05$.

\section{RESULTS}

Over a period of 3 years, 35 patients met the criteria for inclusion. The average age of patients was $52 \pm 13$ years with extremes from 18 to 68 years. There were 23 males and 12 females. Distension of abdomen, abdominal pain and weight loss were the most frequent, observed symptoms in 33 patients (100\%), 26 patients (74.28\%) and 18 patients (51.42\%) respectively. Peritoneal tuberculosis and carcinomatosis were the two most common causes found in 20 patients $(57.14 \%)$ and 13 patients $(37.14 \%)$ respectively.

The appearance of ascites fluid was yellow in 26 patients $(74.28 \%)$ and hemorrhagic in 9 patients $(25.71 \%)$. Ascites was lymphocyte-rich $(>1000 / \mathrm{mm} 3)$ in 19 patients $(54.28 \%)$ and exudative in 33 patients (94.28\%). Ascitic fluid analysis for acid fast bacilli and malignant cytology was negative in all patients. CA-125 was raised in $7(20.0 \%)$ patients, CEA in 4 patients (11.42\%) and CA $19-9$ was raised in 2 patients (5.71\%).The anomalies noted on CT scan, were a omental thickening in 28 cases $(80 \%)$, peritoneal nodules in $7(20 \%)$ patients and the intraabdominal lymph nodes in 13 patients (39.39\%). Ovarian mass was found in 4 patients (11.42\%).

The main clinical, laboratory and CT characteristics are summarized in Table-I On laparoscopy, peritoneal nodules were present in 30 (85.71\%) patients, omental thickening in 26 patients (78.9\%), other associated findings were: adhesions in 30 patients $(85.71 \%)$, peritoneal thickening in 12 patients (36.3\%). Only adhesions were present in 5 patients (15.15\%). The histological diagnosis in these forms was peritoneal tuberculosis in 3 patients, a non-specific inflammation in 2 patients Table-II. Laparoscopic visual diagnosis was in favor of a peritoneal carcinomatosis in 9 cases $(25.71 \%)$, peritoneal tuberculosis in 21 cases (60\%), and nonspecific infection in 5 cases $(14.28 \%)$.

The histological diagnosis was a peritoneal carcinomatosis in $13(37.14 \%)$ patients and peritoneal tuberculosis in 20 (57.1\%) patients and in two patients nonspecific inflammation. The peritoneal carcinomatosis was of ovarian origin in 8 patients, colorectal origin in 3 patients and gall bladder origin in two patients. The sensitivity and specificity of laparoscopic diagnosis in the diagnosis of peritoneal tuberculosis were $78.67 \%$ and $98.6 \%$ respectively and in the diagnosis of peritoneal carcinomatosis were $94.78 \%$ and $72.2 \%$ respectively.

\begin{tabular}{|c|c|c|}
\hline & $\begin{array}{c}\text { Peritoneal tuberculosis } \\
\mathbf{N = 2 0}\end{array}$ & $\begin{array}{c}\text { Peritoneal carcinomatosis } \\
\mathbf{N}=\mathbf{1 3}\end{array}$ \\
\hline Distension of abdomen & $20(100 \%)$ & $13(100 \%)$ \\
\hline Abdominal pain & $17(85 \%)$ & $9(69.23 \%)$ \\
\hline Weight loss & $13(37.14 \%)$ & $5(3.84 \%)$ \\
\hline Ascitic fluid & & $10(28.57 \%)$ \\
\hline Serous & $16(80 \%)$ & $7(20 \%)$ \\
Hemorrhagic & $2(10 \%)$ & $5(14.28 \%)$ \\
Lymphocyte rich & $14(40 \%)$ & \\
\hline CT findings & & $5(14.28 \%)$ \\
\hline Peritoneal nodules & $2(10 \%)$ & $10(76.92 \%)$ \\
Omental thickening & $18(90 \%)$ & $4(30.76 \%)$ \\
Abdominal lymphadenopathy & $9(45 \%)$ & $4(30.76 \%)$ \\
Ovarian mass & 0 & \\
\hline
\end{tabular}

Table I: Clinical, laboratory and CT features of patients according to the two main etiologies. 


\begin{tabular}{|l|c|c|}
\hline & $\begin{array}{c}\text { Peritoneal tuberculosis } \\
\mathbf{N = 2 0}\end{array}$ & $\begin{array}{c}\text { Peritoneal carcinomatosis } \\
\mathbf{N = 1 3}\end{array}$ \\
\hline Peritoneal nodules & $17(85.0 \%)$ & $13(100 \%)$ \\
\hline Peritoneal thickening & $7(35.0 \%)$ & $5(38.46 \%)$ \\
\hline Omental thickening & $15(75.0 \%)$ & $11(84.61 \%)$ \\
\hline Adhesions & $17(85.0 \%)$ & $13(100 \%)$ \\
\hline Only adhesions & $3(15.0 \%)$ & 0 \\
\hline Ovarian mass & 0 & $3(23.07 \%)$ \\
\hline
\end{tabular}

Table II: The laparoscopic findings according to the two main etiologies.

The positive predictive value was $97.3 \%$ and the negative predictive value was $73.7 \%$ for peritoneal tuberculosis and for peritoneal carcinomatosis the positive predictive value was $83.7 \%$ and negative predictive value was $94.87 \%$.

\section{DISCUSSION}

Ascites of unknown origin are dominated by tuberculosis and peritoneal carcinomatosis requiring early diagnosis and care $\mathrm{e}^{1,2}$. The means of imaging (ultrasound, CT scan, MRI) have limitations in the etiological diagnosis of these patients ${ }^{6}$. Several series of literature ${ }^{7,8}$, confirmed the feasibility of laparoscopy in the exploration of the ascites of unknown origin as well as its high sensitivity and specificity.

Three types of lesions are described in peritoneal tuberculosis. Peritoneal granulations are whitish or yellowish, uniform size, the size of a pin head, not exceeding $5 \mathrm{~mm}$. Adhesions result from the Organization of fibrinous exudates, between two peritoneal layers. The inflammatory phenomena manifested by congestion, hypervascularisation and edematous state of peritoneum. In literature, the granulations are the most frequently encountered aspect ( $66 \%$ to $100 \%$ of the cases), adhesions were observed in $13 \%$ to $80 \%$ of cases, and inflammatory phenomena are described in $21 \%$ to $79 \%$ of the cases $^{9}$.

For our patients with peritoneal tuberculosis, the granulations were observed in $85 \%$ of cases, adhesions in $85 \%$ cases and inflammatory phenomena in $35 \%$ of cases. In the peritoneal carcinomatosis, peritoneal implants are generally larger upto 1 $\mathrm{cm}$ and distributed irregularly on the peritoneum, abdominopelvic viscera and diaphragm.

In a series of Barnard et ${ }^{2}$ al involving 90 patients the positive predictive value of the laparoscopy in peritoneal tuberculosis was $85 \%$. Peritoneal biopsy confirmed the diagnosis of tuberculosis and peritoneal carcinomatosis in $98 \%$ and $100 \%$ of the cases respectively.The sensitivity and specificity of visual diagnostic laparoscopic in 176 patients with ascites of undetermined origin,the sensitivity and specificity of diagnostic visual laparoscopic peritoneal tuberculosis were $86 \%$ and $100 \%$ respectively. In the peritoneal carcinomatosis, the sensitivity and specificity were $100 \%$ and $94 \%$, respectively ${ }^{10}$.

Sheth SS et al. ${ }^{7}$ assessed the contribution of laparoscopy in ascites of unknown origin in 70 women. Laparoscopy and peritoneal biopsies have highlighted the diagnosis in $90 \%$ of cases. Nassir et al ${ }^{11}$ assessed the contribution of laparoscopy in exuding ascites of unknown origin in 33 patients. The diagnostic yield of this exploration was $97 \%$.

In our series, sensitivity and specificity of the laparoscopic diagnosis in the diagnosis of peritoneal tuberculosis were $78.67 \%$ and $98.6 \%$ respectively. In the peritoneal carcinomatosis, the sensitivity and specificity were $94.78 \%$ and $72.2 \%$ respectively. This shows that the only visual laparoscopic diagnosis helps to confirm the diagnosis of tuberculosis and eliminate a carcinomatosis in most of cases. Histology allowed confirming the diagnosis of tuberculosis and peritoneal carcinomatosis in all cases.

\section{CONCLUSION}

Laparoscopy with peritoneal biopsy has still got a role in diagnosing the cause of ascites of unknown origin when other modalities of investigations fail to reveal the etiology.

\section{REFERENCES}

1. Hasson, H.M. Open Laparoscopy: A Report of 150 Cases. Journal of Reproductive Medicine 1974;12:234-238.

2. Bedoui, H., Ksantini, R., Nouira, K., et al. Role of Laparoscopic Surgery in the Etiologic Diagnosis of Exudative Ascites: A Prospective Study of 90 Cases. Gastroentérologie Clinique et Biologique 2004;31:1146-1149.

3. Lingenfelser T, Zak J, Marks IN, Steyn E, Halkett J, Price SK. Abdominal tuberculosis: still a potentially lethal disease. Am J Gastroenterol 1993;88:744-50.

4. Sanowski RA. Are there any remaining indications for laparoscopy? Surv Dig Dis 1984;2:115-20.

5. Gandolfi L, Rossi A, Leo P, Solmi L, Muratori R. Indications for laparoscopy before and after the introduction of ultrasonography. Gastrointest Endosc 1985;31:1-3.

6. Parsons, S.L., Lang, M.W. Malignant Ascites: A 2-Year Review from a Teaching Hospital. European Journal of Surgical Oncology 1996; 22: 237-239.

7. Sheth, S.S. The Place of Laparoscopy in Women with Ascites. 
British Journal of Obstetrics and Gynaecology 1989; 96:105-106.

8. Sharma, M.P. and Bhatia, V. Abdominal Tuberculosis. Indian Journal of Medical Research 2004;120:305-315.

9. Singh, M.M., Bhargava, A.N. and Jain, K.P. Tuberculous Peritonitis. The New England Journal of Medicine 1969;281:1091-1094

10. Han, C.M., Lee, C.L., Huang, K.G, et al. Diagnostic Laparoscopy in Ascites of Unknown Origin: Chang Gung Memorial Hospital 20 Year Experience. Chang Gung Medical Journal 2008;31:378-383.

11. Luck, N.H., Khan, A.A., Alam, A., Butt, A.K. and Shafquat, F. Role of Laparoscopy in the Diagnosis of Low Serum Ascites Albumin Gradient. Journal of Pakistan Medical Association 2007;57:3334. 